\title{
EXPLORING THE INCLUSION OF DESIGN FACTORS IN COMPUTER AIDED INVENTING
}

Rosario Vidal ${ }^{1}$, Elena Mulet ${ }^{1}$, Belinda López-Mesa ${ }^{2}$, M. José Bellés ${ }^{1}$ and Graham Thompson ${ }^{3}$

${ }^{1}$ Universitat Jaume I, Castellón, Spain; ${ }^{2}$ Luleå University of Technology, Luleå, Sweden;

${ }^{3}$ UMIST, Manchester, U.K.

Abstract: In this paper we present three experimental research studies that examine the use of different means of expression, the methods used to obtain solutions and additional stimulation; the results of this work can affect the design of future CAI systems.

Key words: CAI, Idea generation, Design teams, Creative problem solving.

\section{INTRODUCTION}

Invention in the initial phases of the design process is one of the most important topics in design engineering and it becomes even more interesting when it is linked to computer systems.

We understand CAI systems to be a valuable aid to designers in creative problem solving and, like other authors, we also believe that there is still a lot to be learnt about how people actually invent. Experimental research can, therefore, provide us with the knowledge needed to design systems for the future.

In this paper we present three experimental research studies, the results of which can have repercussions on future CAI systems. This work examines the use of different means of expression (words, drawings and objects), the 
methods employed to obtain solutions (searches, combinations and transformations) and additional stimulation by means of methods.

\section{EXPERIMENTING WITH DIFFERENT MEANS OF EXPRESSION}

The means used to express ideas plays a number of different roles in the design process. Drawings, as well as acting as a way of communicating and storing the geometric shape, also contribute to other functions by expanding short-term memory, facilitating and verifying design simulation, and stimulating the imagination and creative synthesis ${ }^{1,2,3}$

Some studies reveal that employing the hands and drawings to aid the design process offers very important results that are better than those obtained when the search for solutions is solely a cognitive activity aided by drawings ${ }^{4}$. So much is this so that if the tools developed to aid creative design are to produce an environment that encourages the creativity of the designer, one of the requisites they must satisfy that of using objects during the design process 5 .

In order to observe the influence exerted by the means used to express ideas, three variations of brainstorming involving different means of expression were used in an experiment carried out with Technical Engineering in Industrial Design students from Universitat Jaume I in Castellón, Spain. In all, the study involved 12 groups of 4 people.

The design problem consisted in generating solutions for a drafting table that was to take up as little space as possible when not in use. Each of the twelve groups was given a list with the same initial requirements and at the same time 3 different means of expressing ideas were established; three variations of the means of expression, namely, verbal or sentential, visual and objectual, were therefore utilised.

In the objectual variant, in addition to expressing ideas verbally, they were also constructed using the pieces and tools from 2 sets of Meccano ${ }^{\circledR}$.

The experiments were recorded on video so that the protocol could be obtained and later analysed using the linkography method ${ }^{6}$ but with a certain number of modifications ${ }^{7}$.

The generated ideas were identified so as to allow the design process to be analysed. Here, "generated ideas" is taken to mean any contribution to the formation of a design solution that was communicated in the course of the design process. Ideas are aggregated on a more general level that has been called the global idea and consists in one or more ideas that belong or contribute to the same solution for the design problem. 
From the analysis of the ideas obtained it can be seen that the means of expression used does not give rise to any significant difference in the total number of ideas produced. Nevertheless, significant differences are observed with regard to the type of ideas that are obtained.

The way ideas expressed by means of objects stimulate the generation of later ideas is unlike that employed by ideas that are expressed verbally or by drawings. When the design group uses objects to represent ideas, the percentage of ideas that can become feasible solutions to the problem is seen to be greater. Furthermore, the use of objects is also linked to a stronger tendency to generate subsequent ideas that are related to the most interesting ideas that arose during the design process ${ }^{8}$.

The use of objects leads to the generation of more ideas that are centred on particular aspects of the problem. Moreover, there is a continuous stream of new ideas that build on and improve the previous ones concerning this same aspect. When verbal means are employed the ideas that arise are not clearly focused on any particular aspect of the problem, they are more abstract, the analysis of the problem is less intense or in-depth, and they coevolve with the solution to a lesser degree than is the case when objects are used.

Findings indicate that an interesting approach is to use a system of representation based on 3D manipulation in order to boost the stimulation of invention based on representation by objects. Invention that is stimulated by this means will exert an influence and allow the designer to conduct a more thorough analysis and to gain a deeper understanding of the problem. At the same time co-evolution of the invention process will be greater and it will become easier to obtain possible solutions to the problem.

\section{ANALYSING THE METHODS OF OBTAINING SOLUTIONS}

One of the factors that influence the design process is the method employed to synthesise or to obtain solutions. Computer systems for synthesising or obtaining solutions make use of different techniques to generate solutions to a problem.

Boden sets out two groups of computational models of artificial intelligence for creativity, which is understood to mean the generation of ideas that are both new and valuable ${ }^{9}$. One of them is that of combinational creativity, where the idea that is obtained consists of an unusual combination or association between other familiar ideas. The other one is the exploratorytransformational creativity group, which is based on a vast structured conceptual space. The exploratory creativity models define a conceptual 
space and a set of procedures for moving inside that space and reaching locations in which suitable solutions exist, as is the case of models based on heuristic searches and discovery models. Transformational creativity applications can modify their own rules and include, for example, models based on evolutionary techniques.

Chakrabarti classified the development of computer systems for synthesis in two categories: compositional synthesis and the retrieval of existing designs for them to be modified to fit the requirements ${ }^{10}$. Applying both compositional methods of obtaining solutions and those based on the retrieval and adaptation of cases in a joint or hybrid manner has been proposed as a way of expanding the possibilities offered by these systems ${ }^{11}$.

In order to analyse the effect exerted by applying each method of obtaining solutions, we examined how the design process evolved by analysing the methods employed to obtain solutions during the formation of potential solutions.

One of the methods for studying the evolution of the design is the FBS method, which derives from the design protocol and is used to identify the elements of design by means of functions (F), operation or behaviour (B) and structures $(\mathrm{S})$ at each stage of the design process ${ }^{12,13}$. Function describes the goal of the design, whereas structure describes the solution, and behaviour describes the operation and the change of state of the structure; hence, the design process is expressed as the step from function to structure via its behaviour.

The protocol for the experiment described in the section above was analysed again using the FBS method in order to examine the methods for obtaining solutions. Experimental analysis of the ideas generated in a brainstorming session revealed that the group of designers applied more than one method during the formation of different potential solutions to the problem. For example, they started by conducting one or several isolated searches, these were then combined with one another, then a modification was introduced, and so on. That is to say, each of the potential solutions was obtained by applying the different methods for obtaining solutions in succession and not by utilising just one ${ }^{8}$, which coincides with Chakrabarti's proposal concerning hybrid methods.

The analysis that was conducted showed that obtaining so many and such diverse ideas in such a short time was partly due to the diversity of the methods of synthesis that were applied. The more heterogeneous the methods are, the more potential the solutions will have to resolve problems. These observations imply that the simultaneous use of methods of synthesis based on search techniques, combinations and transformations helps to obtain solutions that resolve the problem. 


\section{EXPERIMENTING WITH ADDITIONAL STIMULI}

The stimulation that arouses the creativity of the designer or the design group is a core issue in current methods of generating ideas. In some approaches this stimulation is essentially achieved using stimuli generated within the actual group itself by means of brainstorming and its variants, and the ideas that are generated play two fundamental roles: they can either be a solution or they can also act as a stimulus in the generation of new ideas. In other methods, such as SCAMPER, Direct Analogy and so on, additional stimuli are provided that arouse individual creativity.

In order to study what happens when additional stimuli are introduced into design groups during the phase in which ideas are generated for a product, an experiment was carried out as part of a joint research study with Luleå University of Technology (Sweden), Universitat Jaume I (Spain) and UMIST (England). The experiment was conducted by Design Engineering $\mathrm{PhD}$ students. Five groups of three people were set up, four of which are discussed in this chapter. The analysis of the results is based on two theories: the innovative-adaptive characteristic theory of methods and the reflective practice theory. This analysis is used here to study the implications they have in the creation of Computer Aided Inventing systems.

To be able to draw conclusions about the effect produced by the additional stimuli that were introduced in the experiment, first we had to identify and catalogue the other stimuli that could affect the way the creative activity progressed. These other stimuli were those that were generated spontaneously within the group and included drawings, sentences, gestures, and so on.

The 17 possible participants met for approximately 40 minutes and were given the initial requirements of the problem together with technical and market data. During this time they read the problem individually and used two different models of the object that was to be redesigned, namely, a tubular map case. Any doubts regarding the initial requirements of the problem were discussed and settled. The goal was to design a tubular map case that allows for one by one extraction and introduction of maps. Each group then went to a different room where they were given precise instructions on how to proceed. The first 5 minutes of the idea creation session had no additional stimuli, but then seven additional stimuli were introduced by means of a computer display every 5 minutes. Two groups were exposed to visual images obtained from the Internet by introducing words related to characteristics linked to the shape and use of the object to be designed. The other two groups were exposed to questions from the 
SCAMPER method. Each visual stimulus contained three images or questions.

Once the protocols have been analysed, one remark that can immediately be applied to the design of CAI systems is that while they are being developed thought must be given as to whether they encourage or hinder the natural mechanisms that designers use to express themselves and what consequences this can have on their creative activity. A particular system could, for example, make it more difficult to perform the gestures required to explain the ideas, and this would hold up the expansion of ideas within the same perspective. Different stimuli bring about different effects in the designer's creative activity and these effects must be taken into account in order to decide what kind of additional stimulus is best suited to a particular situation, if indeed any of them are. The findings from the experiment presented above show that stimuli have an important effect on the design activity, which is comparable to the person's innovative-adaptive characteristic, and that there are stimuli that exert a greater and lesser influence. We also know the types of action (naming, framing, moving and reflecting) that are favoured by the visual stimuli introduced in the experiment and the questions. The stimuli that were measured can be introduced as computer-aided methods, or parts of methods or methodologies, and can also be generated by the computer itself. A stimulus that is not predefined (like that of the images) can be sought automatically on the Internet by the computer. Depending on the type of stimulus we wish to obtain, the computer will need to interact with the designers to a greater or lesser extent before being able to generate those stimuli. For example, in the case of the images that were used to stimulate ideas for the tubular map case, the computer would have to ask the designers for words that describe the shape of the case and how it will be used. Once the words (such as tube, cylinder, tubes, classifier, organiser and so on) have been introduced by the designers, the computer searches for images.

Detailed knowledge of the effects of the stimuli collected in a computer system can substitute the role of a facilitator. The facilitator adapts his or her own experience to each situation and the experience accumulated over the years about design methods enables them to make decisions about the most suitable way of facilitating the group. A thorough study of how the methods affect the design activity is required to make up for the facilitator's adaptability. A good knowledge of the effect of the method and the situation in which that effect is suitable enables the design group to choose the most appropriate stimulus ${ }^{14,15,16}$. 


\section{CONCLUSION}

Experimental research can provide interesting findings that can help to improve CAI systems for the future. From the three experiments that have been commented above we could conclude that it would be advisable to incorporate systems of representation in three dimensions, to apply different methods of synthesis and to include various forms of additional stimuli.

\section{REFERENCES}

1. Ullman, D., Wood, S., et al., 1990, The importance of drawing in the mechanical design process, Computational Graphics. 14(2): 263 - 274.

2. Purcell, A., and Gero, J., 1998, Drawings and the design process, Design Studies 19(4): 389 $-430$.

3. Schütze, M, Sache, P., et al., 2003, Support value of sketching in the design process, Research in Engineering Design. 14: 89 - 97.

4. Candy, L., and Edmonds, E., 1996, Creativity design of the lotus bicycle: implications for knowledge support system research, Design Studies 17(1): 71 - 90.

5. Candy, L., 1997. Computers and creativity support: knowledge, visualization and collaboration. Knowledge-Based Systems. 10(1): 3 - 13.

6. Goldschmidt, G., 1992, Criteria for design evaluation: a process-oriented paradigm, in: Evaluating and Predicting Design Performance. Y. Kalay, ed, John Wiley \& Sons, pp. 67 -79 .

7. Van der Lugt, R., 2000, Developing a graphic tool for creative problem solving in design groups, Design Studies 21(5): 505-522.

8. Vidal, R., Mulet, E. and Gómez-Senent, E., 2004, Effectiveness of the Means of Expression in Creative Problem Solving in Design Groups, Journal of Engineering Design 15(3): 285297

9. Boden, M., 1999, Computer models of creativity, in Handbook of Cretivity, R. Sternberg, Cambridge University Press, pp. 351-372

10. Chakrabarti, A., 2002, Engineering Design Synthesis. Understanding, Approaches and Tools, Springer-Verlag, London.

11. Chakrabarti, A., 2001, Towards hybrid methods for synthesis, ICED'01 13th International Conference on Engineering Design, Imeche, Glasgow

12. Gero, J., 1990, Design prototypes: a knowledge representation schema for design, AI magazine 11(4): 26 - 36

13. Umeda, Y., Takeda, H., et al., 1990, Function, behaviour, and structure, in: Applications of Artificial Intelligence in Engineering,. J. Gero. Berlin, Springer. 1:177-194

14. López-Mesa, B., 2002, Selection and Use of Engineering Design Methods Using Creative Problem Solving Principles, licentiate thesis, Luleå University of Technology, Luleå, Sweden.

15. Mulet, E., R. Vidal, et al., 2003, Influences of representations on designing and implications for computer-based design synthesis, ICED'03 $14^{\text {th }}$ International Conference on Engineering Design, Design Society, Stokholm

16. Mulet, E., 2003, Análisis Experimental y Modelización Descriptiva Del Proceso de Diseño, $\mathrm{PhD}$ thesis, Politechnical of Valencia, Valencia 